\title{
Optimization Method of Security Control Strategy for Dynamic Stability
}

\section{in Interconnected Power Grid}

\author{
Fuqiang $\mathrm{Li}^{1, \mathrm{a}}$, Yuou $\mathrm{Hu}^{1, \mathrm{~b}}$, Zhonghua $\mathrm{He}^{1, \mathrm{c}}$, Yanhong Liang ${ }^{2, \mathrm{~d}}$ \\ ${ }^{1}$ North China Grid Company Limited, Beijing 100053, China \\ ${ }^{2}$ School of Electrical and Electronic Engineering, North China Electric Power University, Baoding \\ 071003, China \\ ali.fuqiang@nc.sgcc.com.cn, ${ }^{b}$ hu.yuou@nc.sgcc.com.cn, ${ }^{c h e . z h o n g h u a @ n c . s g c c . c o m . c n, ~}$ \\ d852678580@qq.com
}

Key words: Dynamic stability; security control strategy; damping torque; torque decoupling; electrical distance

Abstract. It may lead to low frequency oscillation in interconnected power grid if part of the lines of power transmission section are disconnected. Reducing the output of generators in sending end urgently is an effective measurement to restrain oscillation, and different control strategy may lead to different effect. The damping of generators' control system and its electrical distance to the receiving end are the two important factors which impact the effect of generators tripping. The factors above are quantified respectively by damping contribution and voltage phase angle difference of nodes in this paper, and they are weighted to order according to priority of generators tripping. The damping contribution accumulates the effect of damping torque segmentally by decoupling the electromagnetic torque to synchronous torque and damping torque. The actual power grid system of North China is used to verify the correctness of the method. By comparing the existing security control strategy with the result of the proposed method, it shows that the method in this paper can optimize control performance and reduce control cost. Therefore, this method has some practical value.

\section{Introduction}

The scale of national interconnected power grid is increasing, because of the uneven distribution of energy resources and the growing demand for electricity. The transmission of power energy with large capacity at a long distance, can help to administrate power resources across wide areas, but also easily brings about the weak or negative damping low-frequency oscillation between the regional power grids. The fast excitation system with high magnification can effectively improve the static and transient stability of the system, while it may weaken the system damping under heavy load [1], even result as the low-frequency oscillation phenomena.

So far, abundant researches have been conducted by the domestic and foreign scholars, about the mechanism and control strategy of the low frequency oscillation in the interconnected power grid. The low frequency oscillation of multiple national large-scale interconnection projects was investigated in the literature [2], and a valid solution had been summarized, namely, combine the PSS configuration with other measures, like HVDC modulation and Thyristor Controlled Series Capacitor (TCSC). The literature [3] concluded that the negative damping effect caused by Automatic Voltage Regulator (AVR) in excitation system is still main reason for low frequency oscillation in interconnected power system. The low-frequency phenomena was analyzed from the viewpoint of 
damping torques in the literature [4]. And the tests demonstrate that reinforcement of the connection between two bulk power grids is an effective way to mitigate oscillation in system. The literature [5] and [6] studied the calculation method of the synchronous torque and damping torque of the multi-machine power system, besides analyzed the dynamic stability of the system by combining with the modal calculation.

The literatures [7-9] pointed out that the total energy of the system was corresponding to the amplitude in the low frequency oscillation, and further, the components that consumes energy can contribute to converge the oscillation, accordingly possess positive damping, while the ones generating power act as the oscillators whose damping are negative. Actually, a considerable number of units may make a negative contribution to the oscillation attenuation, in the low frequency oscillation of the interconnected system. And unlike the low frequency oscillation caused by the local disturbance source, there exists relatively more units with negative damping. As a result, reducing the output of key units, not removing the above generators completely, should be used to converge the oscillation.

In engineering, appropriate control strategies is usually taken off-line, for those faults arousing probably low frequency oscillation of power system. However, the selection of the withdrawing-units mainly depends on simulation curves and experience, lacking of precise calculation and analysis. From that, it is difficult to adapt to the change and development of the power grid. Therefore, an optimization method for control strategy aimed at the dynamic stability of interconnected power system, was summarized in this paper. The research took into account, not only the damping of control system but also the electrical distance between the units and import network. Then the generators to withdraw can be selected in accordance with priority. The method is proved to be able to optimize the performance of control schemes, reduce the cost as well.

\section{Factors of Dynamic Stability Control}

The Damping of Generator Control System. When the power grid is subjected to disturbance, the generator's PSS, the excitation winding, the damping winding and the mechanical system can provide a certain positive damping. Whereas the excitation system and the speed regulator may be expressed as negative damping in a certain situation, which will weaken the overall damping of the system, as a consequence, interconnected network will oscillate.

Fig.1 shows transfer function schematic of single-machine infinite-bus system, inside, excitation system, speed regulating system and dynamic procedure of PSS, has been considered, in the generator model.

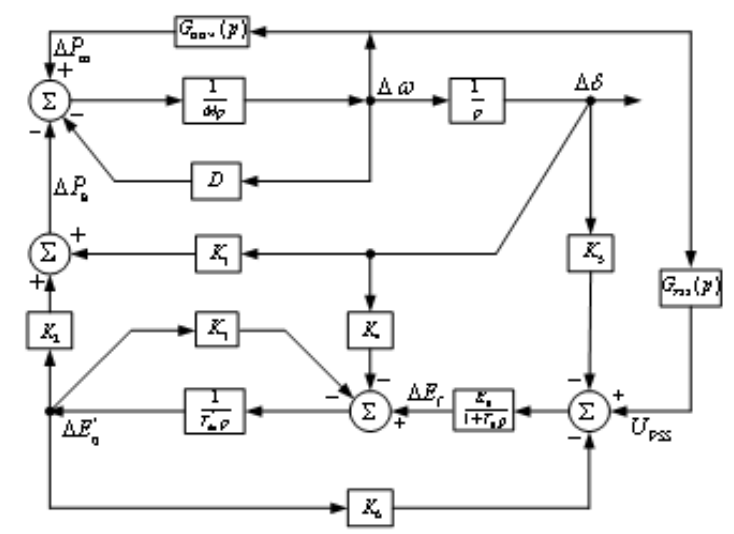

Fig. 1 Transfer function schematic of single machine infinite bus system 
Speed regulating system acts on the rotor motion by changing the mechanical torque [10], and in the low frequency oscillation process of the interconnected system, the frequency of generator rotors fluctuates approximatively around $50 \mathrm{~Hz}$ with a slight deviation of speed amplitude. So that, there won't appear high frequency or low frequency lasting a long time, at the same time, the inertia of speed regulator will delay the response speed of the mechanical power. Therefore, in the above oscillation, as the mechanical power fluctuates slightly, its damping torque acting on the rotor can be neglected.

The excitation system acts on the rotor motion by changing the electromagnetic torque. The change of the electromagnetic torque- $\Delta T_{\mathrm{e}}$ can be decomposed into two components, namely, the synchronous torque and the damping torque, when $\Delta \omega$ is small:

$$
\begin{aligned}
\Delta T_{\mathrm{e}} & =K_{\mathrm{l}} \Delta \delta+K_{2} \Delta E_{\mathrm{q}}^{\prime} \\
& =K_{\mathrm{e}} \Delta \delta+D_{\mathrm{e}} \Delta \omega .
\end{aligned}
$$

In this equation, $\Delta \delta$ : variation of the power angle of generator relative to Steady operating point $-\delta_{\mathrm{o}} ; \Delta \omega$ : changes of rotor speed relative to Steady operating point- $\omega_{0}, K_{\mathrm{e}}$ : the synchronous torque coefficient ; $D_{\mathrm{e}}$ : the damping torque coefficient.

At the moment, the synchronous torque affected mainly the oscillation frequency while the damping one relates directly to the trend of the oscillation. When $D_{\mathrm{e}}>0$, the positive damping torque component in phase with $\Delta \omega$, from electromagnetic torque, is helpful to suppress the low frequency oscillation [11]. In Fig. 1 , the parameter $K_{5^{-}}$partial derivative of generators terminal voltage to power angle, may be negative as the generator is under heavy-loading. And when $K_{5}<0, \Delta T_{\mathrm{e}}$ locates at the III, IV quadrant of the phase plane $\Delta \omega-\Delta \delta$, that is, damping of the excitation system is negative.

In the case of large-capacity power transmission across long distance, the power angle of the generator at the sending end is relatively large. Especially, after the transmission line trips, the electrical distance between the two sides increases, and the power angle of the generator at the sending end also rises, thus, the excitation system of the generator may provide a negative damping. As a conclusion, with regard to the low frequency oscillation of the interconnected power grid, the excitation system is the main reason for the deterioration of massive sending units damping.

The Electrical Distance between the Generators and Import Network. The paper [12] pointed out that, under the new network structure after faults, the power output of those large units far away from the import system, should be reduced rapidly. Furthermore, control effects were compared, when to trip different units at near-end or far-end, in the four-machine infinite system.

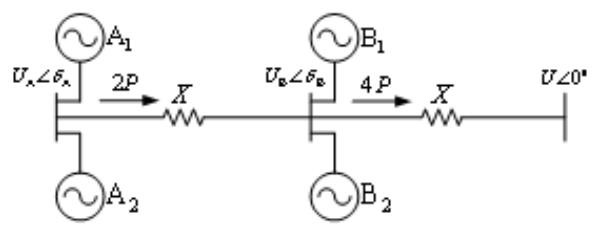

Fig. 24 machines infinite bus system

Assume that the active power of the four units are $\mathrm{P}$, the reactance of the two power transmission section both are $\mathrm{X}$, every bus voltage is 1 . When to remove unit $\mathrm{B}_{1}$, the voltage phase of bus-B and bus-A are expressed as Eq. (2): 


$$
\begin{aligned}
& \delta_{\mathrm{B}}=\arcsin (3 P X) \\
& \delta_{\mathrm{A}}=\arcsin (2 P X)+\delta_{\mathrm{B}} .
\end{aligned}
$$

When to remove unit $A_{1}$, express them as:

$$
\begin{aligned}
& \delta_{\mathrm{B}}=\arcsin (3 P X) \\
& \delta_{\mathrm{A}}=\arcsin (P X)+\delta_{\mathrm{B}} .
\end{aligned}
$$

Obviously, the change of voltage phase angle of bus-B when tripping unit $\mathrm{A}_{1}$, is the same with that when tripping unit $B_{1}$, so the two kinds of control measures have the same effects on the damping of units connected with bus-B. On the other hand, voltage phase angle of bus-A decreased more, when unit $A_{1}$ is tripped, than unit $B_{1}$ done, thus tripping unit $A_{1}$ influence much the damping of units connected with bus-A.

In summary, the power flow on many transmission section would drop if made the distal unit off of operation, and the damping of other units enhance obviously greater. Therefore, the electrical distance between generators and import network is another important factor that affects to select tripping-units, so as to stay dynamically stable.

\section{Generators Tripping Priority}

Damping Contribution Index. From the above analysis, it can be known that the damping state of generator in the low frequency oscillation is related to several factors, including the structure and parameters of the network, the operating condition and the parameters of the generator control system. In the oscillation, some units may show positive damping, consuming the oscillation energy, while some have negative damping, providing energy to oscillation. At the same time, the negative damping effect in different ways [13-14]. When two units locate nearly, removing one with low damping from grid can get a better control effect. In a word, we should assess the damping contribution of units when making the control strategy, and the units providing larger negative damping should be tripped first.

As Figure 1 shown, due to $K_{1}>0$, in this path, a component in phase with $\Delta \delta$ appears, which is only related to the synchronous torque; Generally, $K_{2}$, a real number is greater than 0 , so ${ }^{\Delta E_{\mathrm{q}}^{\prime}}$ can be decoupled into the superposition of the synchronous torque and damping torque:

$$
\Delta E_{\mathrm{q}}^{\prime}=K_{\mathrm{e}} \Delta \delta+D_{\mathrm{e}} \Delta \omega
$$

The parameters in Eq. (4) stand for integrated damping involving the excitation windings, excitation system and PSS. And without regard for the damping of speed regulator, the phase relationship between $D_{\mathrm{e}} \Delta \omega$ and $\Delta \omega$, reflects the overall damping properties of the unit control system. Besides, the synchronous torque component in Eq. (4), independent of the low frequency oscillation, can be decoupled away, thereby, cumulate effect of the damping torque separately.

The following quantization expression defines the damping contribution of generator from $t_{1}$ to $t_{2}$ :

$$
\int_{t=t_{1}}^{t=t_{2}}\left(D_{\mathrm{e}} \Delta \omega\right) \Delta \omega d t
$$


If the result is negative, it shows that the unit is in a negative damping state during the period of $t_{1}$ to $t_{2}$, and the higher absolute value of the negative one is, the more key the unit is, to the oscillation, which accordingly should be given first.

Calculation of Damping Contribution. The damping torque coefficient of the generator is difficult to obtain by simple calculation. According to the Eq. (3), damping torque and synchronous torque can be decoupled with identification parameters- $D_{\mathrm{e}}$ and $K_{\mathrm{e}}$ in the matrix form of the least square method. Supposing that the amplitude of the oscillation was slight, the synchronous torque coefficient and the damping torque coefficient of the generator could be considered as constants [15-16]. On this basis, divide data in relation to the vibration equidistantly, calculate the damping contribution of generator by means of segmentation identification and accumulation by section. For example, to calculate the electromagnetic damping, setting the sampling interval of the data as $20 \mathrm{ms,}$ then identify every unit of parameters $\left[\Delta E_{q}^{\prime}, \Delta \delta, \Delta \omega\right]$ as every unit data includes adjacent 10 sets of one. The matrix form of the least squares method is described as Eq. (6):

$$
B=\left(X^{T} X\right)^{-1} X^{T} Y
$$

Every matrix means as show below:

$$
X=\left[\begin{array}{cc}
\Delta \delta_{1} & \Delta \omega_{1} \\
\Delta \delta_{2} & \Delta \omega_{2} \\
\vdots & \vdots \\
\Delta \delta_{10} & \Delta \omega_{10}
\end{array}\right] \quad Y=\left[\begin{array}{c}
\Delta E_{\mathrm{q} 1}^{\prime} \\
\Delta E_{\mathrm{q} 2}^{\prime} \\
\vdots \\
\Delta E_{\mathrm{q} 10}^{\prime}
\end{array}\right] \quad B=\left[\begin{array}{l}
K_{\mathrm{e}} \\
D_{\mathrm{e}}
\end{array}\right]
$$

Since voltage behind direct-axis sub-transient reactance of a synchronous generator is unable to gain directly, generators terminal voltage $\dot{U}$, terminal current $\dot{I}$, stator resistance Ra, direct-axis transient reactance $X_{\mathrm{d}}^{\prime}$ and quadrature-axis synchronous reactance $\mathrm{X}_{\mathrm{q}}$ are be used into the calculation, as Eq. (8):

$$
\left\{\begin{array}{l}
\dot{E}_{\mathrm{Q}}=\dot{U}+\dot{I} R_{\mathrm{a}}+j \dot{I} X_{\mathrm{q}}=E_{\mathrm{Q}} \angle \delta \\
\dot{E}^{\prime}=\dot{U}+j \dot{L} X_{\mathrm{d}}^{\prime}=E^{\prime} \angle \delta^{\prime} \\
E_{\mathrm{q}}^{\prime}=E^{\prime} \cos \left(\delta-\delta^{\prime}\right)
\end{array}\right.
$$

In Fig. 1, the changes of every electrical quantity all are relative to the steady operation point. Once the power grid is subjected to any large disturbance, every generator will run on a new stable state from the previous one, so that steady state value applied should be corresponding to the time where it belongs, while seeking $\Delta E_{\mathrm{q}}^{\prime} 、 \Delta \delta$ and $\Delta \omega$. Further, collect the peak and valley values of electric-quantity at each oscillation cycle, and then curve-fit the envelopes of the peak and valley values by using cubic spline interpolation method, thereby select the median of two envelopes at the same time as the steady-state operating point.

Electrical Distance Indicator and Generator Tripping Priority. On one hand, the voltage phase angle of bus decreases in the transmission direction of active power. Therefore, that means there was a large difference between the voltage phase angle of bus which generator connects with, and one of 
import grid, if they were away from each other. On the other hand, electrical distance would generally change since the grid structure changed after faults. Consequently, apply the above difference to measure the electrical distance, as using voltage phase angle of the high-voltage-side bus connecting generators. In addition, the method for steady-state value is the same with the last section.

Orthogonalize the damping contribution and the electrical distance index, and change into per unit values, so that the values are between $0 \sim 1$. Putting the same weight 0.5 on the two variables, after weighting, this is the priority of the generator. So that, trip units in accordance with the priority when to take control strategy.

\section{Cases}

Accuracy of the Damping Contribution Index. Firstly, the accuracy of the damping contribution index is verified. Taking the North China Grid as an example, weaken the damping of the units in plant by modifying the parameters of the MengZhun control system and reactance of the plant outlet. Now, put a disturbance on the outlet from this plant, after that, there units become an oscillator, which brings the low-frequency oscillation into MengXi power grid. The waveforms of damping contribution of units in MengZhun and other power plants are shown in Figure 3:

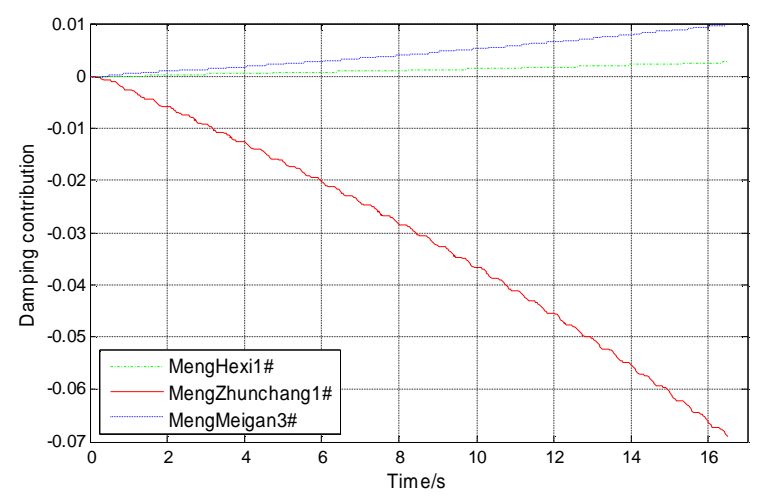

Fig. 3 Waveform of damping contribution

As shown as Fig.3, significantly, the absolute value of damping contribution of MengZhun was greater than that of other units, and the damping contribution during $16 \mathrm{~s}$ accumulation was up to 0.068. Meanwhile, the remaining units with positive damping all consumed energy of the oscillation as the absolute values of damping contribution were generally small. For instance, the damping contribution of accumulated MeiJian 3\# during $16 \mathrm{~s}$ is 0.0095 and that of $\mathrm{HeXi} 1$ \# is 0.0022 .

It can be seen that the damping contribution not only can reflect the damping property of the unit, but also can accurately quantify the importance of each unit in the oscillation, and clearly give the key units to be focus on.

The line HanHai-GuYuan fault. Under the operational mode of MengXi sending out 4250MW, setting N-2 fault on the line HanHai-GuYuan, would lead that MengXi grid increasingly oscillated and failed to be stable, with oscillation frequency $0.313 \mathrm{~Hz}$, and damping ratio -0.015 . In this case, take the control measures of North China as following, removing HeXi units 1\#, 2\# as well as DaQi $5 \#$, namely, totally $1530 \mathrm{MW}$ power to be tripped.

In the absence of control measures, calculate oscillation contributions of all of units in MengXi that were able to be configured security and stability control measures. The Table 1 shows the results lasting $16 \mathrm{~s}$ in length. 
Tab.1 damping contribution of generator in oscillation

\begin{tabular}{|c|c|c|c|}
\hline generator & $\begin{array}{c}\text { Damping } \\
\text { contribution }\end{array}$ & $\begin{array}{c}\text { Electrical } \\
\text { distance }\end{array}$ & $\begin{array}{c}\text { Generator } \\
\text { tripping } \\
\text { Priority }\end{array}$ \\
\hline MengBulian 1\# & -0.8186 & 60.8 & 0.818182 \\
\hline MengHexi 1\# & -0.5668 & 56.6 & 0.541128 \\
\hline MengHexi 2\# & -0.5563 & 56.6 & 0.537536 \\
\hline MengFenghuang 2\# & 0.6429 & 64.8 & 0.5 \\
\hline MengDengkou 1\# & 0.599 & 63.7 & 0.465019 \\
\hline MengJingtai 1\# & -0.6034 & 53.8 & 0.426377 \\
\hline MengMeijian 4\# & -0.546 & 54.2 & 0.424921 \\
\hline MengZhunchang1\# & -0.4643 & 54.1 & 0.392425 \\
\hline MengZhunchang 3\# & -0.3304 & 54.1 & 0.346616 \\
\hline MengMeijian 3\# & -0.3015 & 54.2 & 0.341275 \\
\hline MengZhunchang 2\# & -0.2898 & 54.1 & 0.332726 \\
\hline MengDaqi 6\# & 0.0806 & 56.4 & 0.310553 \\
\hline MengDaqi 5\# & 0.084 & 56.4 & 0.309389 \\
\hline MengZhunchang 4\# & -0.1528 & 54.1 & 0.285857 \\
\hline
\end{tabular}

Obviously, at this operation mode, unit Bulian 1\#, Hexi 1\# and 2\# influenced much more than others on the low frequency oscillation.

After the implementation of the existing scheme in North China, the damping ratio of the low frequency oscillation reached 0.016. Remove more units gradually, according to the order of the damping contribution in table 1 . Then the damping ratio can be the same as that of the original scheme after decreasing the power output up to $1160 \mathrm{MW}$, including Buljan 1\# -660MW, Hexi 1\#-500MW, totally less 370MW than the original one, generally accounting for $24.2 \%$. The active power on the lines Fengquan-Wanquan in delivery section were shown in figure 4, when original scheme and improved one were separately implemented.

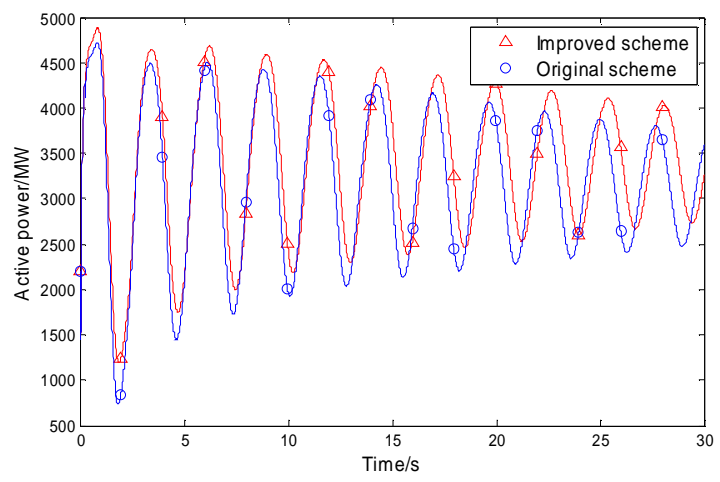

Fig. 4 Comparison chart of power in delivery section

The picture above indicates that the average power in delivery section was about 3080MW if applying the original scheme, while 3320MW when applying the improved scheme. So, the conclusion could be drawn from the results described above, it is to say, this method can optimize the control strategy by reducing the shortage of power that was due to suppress oscillation.

The Line Yongshengyu-Fengquan Fault. When to overhaul one of three transmission lines 
connected Yongshengyu with Fengquan, the power flow is 4724MW in Hu-Feng delivery section, which consists of Wuchuan-Chayouzhong, Qixiaying-Hanhai and Yongshengyu-Fengquan). For another, after developing N-2 fault on the other two lines, the Mengxi network went into increasing oscillation, with oscillation frequency $0.33 \mathrm{~Hz}$, damping ratio -0.0023 . In the control scheme here, total 1560MW power had been tripped, comprising unit Hexi 1\#, Meijian 3\#, and 4\# and unit 1\# in Jingtai.

According to the method proposed in this paper, in the absence of any measures, calculate oscillation contribution index of controllable units. Results are shown in Table 2.

Tab.2 Damping Contribution of generator in oscillation

\begin{tabular}{|c|c|c|c|}
\hline generator & $\begin{array}{c}\text { Damping } \\
\text { contribution }\end{array}$ & $\begin{array}{c}\text { Electrical } \\
\text { distance }\end{array}$ & $\begin{array}{c}\text { Generator } \\
\text { tripping } \\
\text { Priority }\end{array}$ \\
\hline MengBulian 1\# & -0.6378 & 55.4 & 0.777778 \\
\hline MengHexi 1\# & -0.6003 & 50.9 & 0.48218 \\
\hline MengHexi 2\# & -0.5965 & 50.9 & 0.480374 \\
\hline MengFenghuang 2\# & 0.4144 & 58.3 & 0.45679 \\
\hline MengMeijian 4\# & -0.319 & 52 & 0.416409 \\
\hline MengDengkou 1\# & 0.2902 & 56.6 & 0.410871 \\
\hline MengZhunchang1\# & -0.2974 & 51.8 & 0.393799 \\
\hline MengJingtai 1\# & -0.2641 & 51.6 & 0.36563 \\
\hline MengZhunchang 2\# & -0.2077 & 51.8 & 0.351174 \\
\hline MengZhunchang 3\# & -0.1795 & 51.8 & 0.337774 \\
\hline MengMeijian 3\# & -0.1257 & 52 & 0.324554 \\
\hline MengDaqi 6\# & 0.0654 & 51.2 & 0.184362 \\
\hline MengDaqi 5\# & 0.0691 & 51.2 & 0.182603 \\
\hline
\end{tabular}

Table 2 shows, it was Buljan 1\#, Hexi 1\# and 2\# that the damping contribution index were larger. After the existing control scheme, damping ratio reached 0.017 , while it could be achieved by tripping only 1060MW generator output according to the range of damping contribution index. Practically speaking, 660MW was removed from the unit Bulian 1\#, 400MW from Hexi 1\#, and demonstrably tripping power had been gotten down 500MW, accounting for $32 \%$ of that while taking original measure. Figure 5 shows the power curves in delivery section after two kinds of tripping measures.

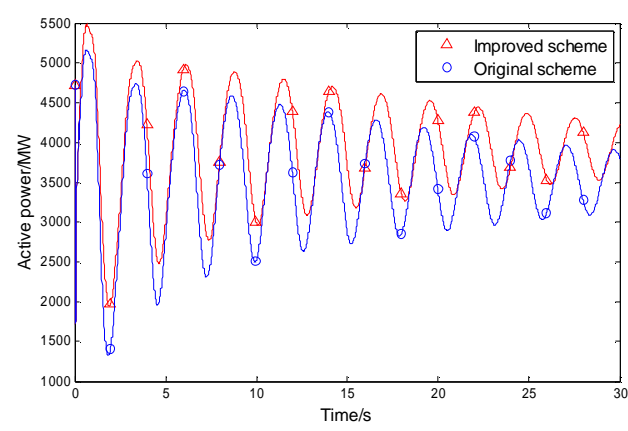

Fig. 5 Comparison chart of power in delivery section 
It can be seen in the graph 5 that the average power after the original scheme is about 3493MW, while it is $3890 \mathrm{MW}$ after taking improved measures, thereby, the control cost has been greatly cut down. So, this method is competent to providing a rigorous data analysis for the off-line scheme. Moreover, it can better keep up the changes of the fault form, the parameters and the structure of the network, while applied to monitor and control the vibration on-line. Thus the system damping can be improved to the most degree in accordance with tripping priority based on the real-time data.

\section{Conclusions}

In brief, under the background where the grid structure, parameters and operation conditions and other factors are changeable, a method has been put forward in this paper, which applies into formulating control strategy for a low-frequency oscillation of interconnected power grid, and further optimizes the effect of security and stability control. Specifically, to begin with, based on the analysis of the negative damping mechanism of the generator control system, the damping contribution index has been presented, that can accurately quantify the cumulative effect of the generator damping torque. Then considering that the electrical distance between the generator and the import network act on control effect, formulate priority index about tripping units. Furthermore, it is turn out that take measures according to previous priority can help to reduce the control cost and obtain good control effect, via the simulation results of the North China power grid. So the proposed method can be applied to work out off-line schemes and monitor oscillations on-line.

\section{References}

[1] Yixin NI, Shousun CHEN, Baolin ZHANG, et al. Theory and Analysis on Dynamic Power System [M]. Beijing: Tsinghua University Press, 2002: 235-266, In Chinese.

[2] Fang ZHU, Yong TANG, Dongxia ZHANG, et al. Study on Dynamic Stability Problems of AC Interconnected Area Power Grids in China and their Solutions [J]. Power System Technology, 2004, 28(15), 1-5, In Chinese.

[3] Fang ZHU, Hongguang ZHAO, Zenghuang LIU, et al. The Influence of Large Power Grid Interconnected on Power System Dynamic Stability [J]. Proceedings of the CSEE, 2007, 27(1), 1-7, In Chinese.

[4] Yixin YU, Peng LI, et al. The Impact of Weak Interconnection of Bulk Power Grids to Damping and Dynamic Stability of Power Systems [J]. Proceedings of the CSEE, 2005, 25(11), 6-11, In Chinese.

[5] Shaltout A A, Abu Al-Feilat K A, Damping and Synchronizing Torque Computation in Multi-machine Power Systems [J]. IEEE Transactions on Power Systems, 1992, 7(1), 280-286.

[6] Abu Al-Feilat K A, Younan N, Grzybowski S. Estimating the Synchronizing and Damping Torque Coefficients Using Kalman Filtering [J]. Electric Power Systems Research, 1999, 52(1), 145-149.

[7] Lei CHEN, Yong MIN, Wei HU. Low Frequency Oscillation Analysis and Oscillation Source Location Based on Oscillation Energy, part one: mathematical foundation and energy flow computation [J]. Automation of Electric Power Systems, 2012, 36(3), 22-27, In Chinese. 
[8] Lei CHEN, Yiping CHEN, Yong Min. Low Frequency Oscillation Analysis and Oscillation Source Location based on Oscillation Energy, part two: method for oscillation source location and case studies [J]. Automation of Electric Power Systems, 2012, 36(4), 1-5, In Chinese.

[9] Lei CHEN, Yong MIN, Wei HU. An Energy-based Method for Location of Power System Oscillation Source [J]. IEEE Transactions on Power Systems, 2012, 28(2), 828-836.

[10] Guanhong WANG, Xiangyu TAO, Wenfeng LI, et al. Influence of Turbine Governor on Power System Dynamic Stability [J]. Proceedings of the CSEE, 2008, 28(34), 80-86, In Chinese.

[11] Marshall W K, Smolinski W J. Dynamic Stability Determination by Synchronizing and Damping Torque Analysis [J]. IEEE Transactions on Power Apparatus and Systems, 2007, 92(4), 1239-1246.

[12] Haitao YANG, Maosheng DING, Xinli SONG. Analysis on Power System Dynamic Stability Mechanisms and Stabilizing Measures [J]. Automation of Electric Power Systems, 2008, 7(25), 35-39, In Chinese.

[13] Wenfeng LI, Jianbo GUO, Ying LI, et al. Power System Oscillation Analysis and Oscillation Source Location based on WAMS, part 1: method of cutset energy [J]. Proceedings of the CSEE, 2013, 33(25), 41-46, In Chinese.

[14] Wenfeng LI, Ying LI, Xiaoxin ZHOU, et al. Power System Oscillation Analysis and Oscillation Source Location based on WAMS, part 2: method of torques decomposition [J]. Proceedings of the CSEE, 2013, 33(25), 47-53, In Chinese.

[15] Renmu HE, Feng SHEN, Dong HAN, et al. The Study of Generator Excitation System Modeling and Parameters Estimation [J]. Power System Technology, 2007, 31(14): 62-67, In Chinese.

[16] Maohai WANG, Dengfeng GUO, Changming JIANG. An Analysis Method to Evaluate Damping Characteristics of Excitation Systems in Low Frequency Oscillation Process [J]. Automation of Electric Power Systems, 2013, 37(4), 47-50, In Chinese. 